\title{
Efficacy and Safety of First-Line Necitumumab Plus Gemcitabine and Cisplatin Versus Gemcitabine and Cisplatin in East Asian Patients with Stage IV Squamous Non-small Cell Lung Cancer: A Subgroup Analysis of the Phase 3, Open-Label, Randomized SQUIRE Study
}

\author{
Keunchil Park, MD, PhD ${ }^{1}$ \\ Eun Kyung Cho, MD, PhD² \\ Maximino Bello, $\mathrm{MD}^{3}$ \\ Myung-Ju Ahn, MD, PhD' \\ Sumitra Thongprasert, $\mathrm{MD}^{4}$ \\ Eun-Kee Song, MD, PhD ${ }^{5}$ \\ Victoria Soldatenkova, MSc ${ }^{6}$ \\ Henrik Depenbrock, MD ${ }^{6}$ \\ Tarun Puri, MD \\ Mauro Orlando, MD
}

\section{Purpose}

The phase 3 randomized SQUIRE study revealed significantly longer overall survival (OS) and progression-free survival (PFS) for necitumumab plus gemcitabine and cisplatin (neci+GC) than for gemcitabine and cisplatin alone (GC) in 1,093 patients with previously untreated advanced squamous non-small cell lung cancer (NSCLC). This post hoc subgroup analysis assessed the efficacy and safety of neci+GC among East Asian (EA) patients enrolled in the study.

\section{Materials and Methods}

All patients received up to six 3-week cycles of gemcitabine (days 1 and 8, 1,250 mg/m²) and cisplatin (day $1,75 \mathrm{mg} / \mathrm{m}^{2}$ ). Patients in the neci+GC arm also received necitumumab (days 1 and $8,800 \mathrm{mg}$ ) until disease progression or unacceptable toxicity. Hazard ratios (HRs) and 95\% confidence intervals (Cls) were estimated from stratified Cox proportional hazards models.

\section{Results}

In EA patients, there were improvements for neci+GC $(n=43)$ versus $\mathrm{GC}(\mathrm{n}=41)$ in OS (HR, $0.805 ; 95 \% \mathrm{Cl}, 0.484$ to 1.341 ) and PFS (HR, 0.720; 95\% Cl, 0.439 to 1.180), consistent with the results for non-EA patients observed in the present study. The overall safety data were consistent between EA and non-EA patients. A numerically higher proportion of patients experienced serious adverse events (AEs), grade $\geq 3 \mathrm{AEs}$, and $A E s$ with an outcome of death for neci+GC versus GC in EA patients and EA patients versus non-EA patients for neci+GC.

\section{Conclusion}

Although limited by the small sample size and post hoc nature of the analysis, these findings are consistent with those of the overall study and suggest that neci+GC offers a survival advantage and favorable benefit/risk for EA patients with advanced squamous NSCLC.

\section{Key words}

Non-small-cell lung carcinoma, Cisplatin, East Asian, Gemcitabine, Necitumumab, Epidermal growth factor receptor
Correspondence: Keunchil Park, MD, PhD Division of Hematology/Oncology, Department of Medicine, Samsung Medical Center, Sungkyunkwan University School of Medicine, 81 Irwon-ro, Gangnam-gu, Seoul 06351, Korea

Tel: 82-2-3410-3459

Fax: 82-2-3410-1754

E-mail:kpark@skku.edu

Received September 2, 2016

Accepted December 14, 2016

Published Online January 6, 2017 


\section{Introduction}

Squamous cell carcinoma of the lung is the second most common histologic subtype of non-small cell lung cancer (NSCLC), after adenocarcinoma, accounting for 30\% of NSCLC cases [1,2]. Advances have been made in the firstline treatment of nonsquamous NSCLC, especially adenocarcinoma, with the advent of better tolerated chemotherapeutic agents (e.g., pemetrexed) and targeted agents (e.g., bevacizumab, epidermal growth factor receptor [EGFR] tyrosine kinase inhibitors [TKIs], and anaplastic lymphoma kinase inhibitors) $[1,3]$. However, clinical trials evaluating these agents failed to show any benefit for patients with squamous NSCLC, and the standard first-line treatment for such patients is still conventional platinum-based doublet chemotherapy $[1,2]$. One of the few treatments that has shown improved overall survival (OS) in patients with squamous NSCLC is cetuximab, a chimeric EGFR antibody. The phase 3 FLEX (First-Line ErbituX in lung cancer) study showed that combining cetuximab with cisplatin and vinorelbine significantly improved OS in patients with EGFR-expressing advanced NSCLC, in particular, in patients with squamous NSCLC (hazard ratio [HR], 0.80; 95\% confidence interval [CI], 0.64 to 1.00) [4]. Although the FLEX study did not lead to the regulatory approval of cetuximab for NSCLC [5], it provided evidence that anti-EGFR monoclonal antibodies might be of value in the treatment of squamous NSCLC. As EGFR protein overexpression and high EGFR gene copy number are frequently observed in patients with squamous NSCLC $[6,7]$, investigation of other agents that target the EGFR may yield new treatment options for squamous NSCLC.

Necitumumab is a second-generation, recombinant, human immunoglobulin G1 EGFR monoclonal antibody that binds the EGFR with high affinity, thereby preventing natural ligand activation $[8,9]$. The multinational, open-label, phase 3 , randomized SQUIRE (SQUamous NSCLC treatment with the Inhibitor of EGF REceptor) study compared necitumumab plus gemcitabine and cisplatin (neci+GC) with gemcitabine and cisplatin alone (GC) in 1,093 patients with previously untreated advanced squamous NSCLC [9]. In this study, OS, the primary endpoint, was found to be significantly longer in the neci+GC arm than the GC arm, with a median OS of 11.5 months being observed in the neci+GC arm and 9.9 months in the GC arm (stratified HR [neci+GC vs. GC], $0.84 ; 95 \% \mathrm{CI}, 0.74$ to $0.96 ; \mathrm{p}=0.01$ ). In addition, progression-free survival (PFS) was significantly longer in the neci+GC arm than the GC arm.

Substantial variations in tumor biology and the efficacy and toxicity of anticancer agents may exist between patients of different ethnicities and have been reported between East
Asian (EA) and Caucasian patients [10,11]. For example, patients of EA ethnicity with advanced nonsquamous NSCLC are more likely to have activating EGFR mutations than Caucasian patients and are therefore more likely to respond to treatment with the EGFR TKI gefitinib [12,13]. In addition, clinical trials of chemotherapy regimens have commonly found higher incidences of toxicities such as grade 4 neutropenia in Japanese study populations than in largely Caucasian study populations [14]. Therefore, this post hoc subgroup analysis was conducted to assess the efficacy and safety of neci+GC in EA patients enrolled in the SQUIRE study. The efficacy and safety results for the EA patients are presented alongside those for the non-EA patients enrolled in the study.

\section{Materials and Methods}

\section{Study design}

The SQUIRE study design has been published in detail elsewhere [9]. The study was a multicenter, open-label, randomized phase 3 study comparing neci+GC with GC in patients with previously untreated advanced squamous NSCLC. The study was conducted in 26 countries, including five countries in East Asia (Philippines, Republic of Korea, Singapore, Taiwan, and Thailand). The study protocol was approved by the ethics committees of the participating sites and conducted in accordance with the Declaration of Helsinki, Good Clinical Practice Guidelines, and applicable local regulations. All patients provided written informed consent before study entry. The study was registered at http:// www.clinicaltrials.gov (NCT00981058).

\section{Study population}

The main inclusion criteria were as follows: age $\geq 18$ years; histologically or cytologically confirmed stage IV (according to the American Joint Committee on Cancer staging manual, seventh edition [15]) squamous NSCLC; Eastern Cooperative Oncology Group (ECOG) performance status of 0-2. The main exclusion criteria were as follows: previous chemotherapy for advanced NSCLC; chest irradiation in the 12 weeks before randomization; peripheral neuropathy of grade 2 or worse (graded according to the National Cancer Institute Common Terminology Criteria for Adverse Events [NCI CTCAE], ver. 3.0). More detailed information regarding the eligibility criteria are published elsewhere [9]. 


\section{Randomization and masking}

Patients were randomly assigned (1:1) centrally to neci+ GC or GC. Randomization was stratified by ECOG performance status (0-1 vs. 2) and geographical region (North America, Europe, Australia vs. South America, South Africa, India vs. East Asia). Physicians and patients were not masked to treatment assignment because the expected occurrence of acne-like rash (a known class effect of EGFR antibodies) would have unmasked most patients and investigators to treatment.

\section{Treatment protocol}

Patients in the neci+GC and GC arms received up to six 3 -week cycles of gemcitabine $1,250 \mathrm{mg} / \mathrm{m}^{2}$ administered intravenously over 30 minutes on days 1 and 8 , and cisplatin $75 \mathrm{mg} / \mathrm{m}^{2}$ administered intravenously over 120 minutes on day 1 (induction phase). During the induction phase, patients in the neci+GC arm also received $800 \mathrm{mg}$ necitumumab administered intravenously over a minimum of 50 minutes on days 1 and 8 , before gemcitabine administration. At the end of the induction phase, patients in the neci+GC arm who had not progressed continued to receive singleagent necitumumab on the same treatment schedule until radiographic documentation of disease progression, unacceptable toxicity, or withdrawal of consent (continuation phase).

\section{Assessment of EGFR expression}

Patients were not selected for the study based on the level of EGFR expression; however, availability of tumor tissue was an eligibility criterion allowing exploratory biomarker analyses. Tumor EGFR protein expression was assessed by immunohistochemistry with the EGFR PharmDx Kit (Dako, Glostrup, Denmark). The level of EGFR expression was classified by immunohistochemistry score (H-score), on a scale of 0 to 300 , as previously described $[6,16]$.

\section{Outcome measures}

Efficacy outcomes were OS (primary outcome of the overall study), PFS, time-to-treatment failure (TTF), objective response rate (ORR), and disease control rate (DCR). OS was defined as the time from randomization until death from any cause. PFS was defined as the time from randomization until the first radiographic documentation of objective progression or death from any cause. TTF was defined as the time from randomization until the first radiographic documentation of progressive disease, death from any cause, discontinuation of treatment for any reason, or initiation of new anticancer therapy. The ORR was defined as the proportion of patients with a best response of complete response (CR) or partial response (PR). The DCR was defined as the proportion of patients with a best response of $\mathrm{CR}, \mathrm{PR}$, or stable disease.

Safety outcomes were adverse events (AEs), which were coded according to the Medical Dictionary for Regulatory Activities ver. 16.0, and graded according to the NCI CTCAE ver. 3.0. AEs of interest were defined based on the known safety profiles of other EGFR antibodies and previous clinical experience with necitumumab, gemcitabine, and cisplatin [9].

\section{Statistical analysis}

This was a post hoc subgroup analysis of the EA patients enrolled in the SQUIRE study. The results for the EA patients are presented alongside those for the non-EA patients enrolled in the study. This presentation is intended as a descriptive illustration of summary data between the two subpopulations and is not a formal comparison. Efficacy was assessed in the intention-to-treat (ITT) population, which comprised all patients who were randomized to treatment. Safety was assessed in the safety population, which comprised all patients who were randomized to treatment and received at least one dose of study medication. Exposure to necitumumab and chemotherapy was reported as the median number of cycles (interquartile range [IQR]) and relative dose intensity (actual dose delivered as a percentage of planned dose). OS, PFS, and TTF were estimated using the Kaplan-Meier method and were compared between treatment arms using the log-rank test. HRs and 95\% CIs for neci+GC versus GC were estimated from stratified Cox proportional hazards models. Post-study systemic therapy was summarized as the number and percentage of patients using therapy. AEs were summarized as the number and percentage of patients reporting each event. Analyses were performed using SAS ver. 9.1.3 (SAS Institute Inc., Cary, NC).

\section{Results}

\section{Patient disposition}

Of the 1,093 patients enrolled in the SQUIRE study, 84 were from East Asia (Republic of Korea, 47; Philippines, 20; Thailand, 9; Taiwan, 5; Singapore, 3) and constituted the EA subpopulation. The remaining 1,009 patients constituted the non-EA subpopulation. In the EA subpopulation, 84 patients were randomized to treatment (neci+GC, 43; GC, 41) and 


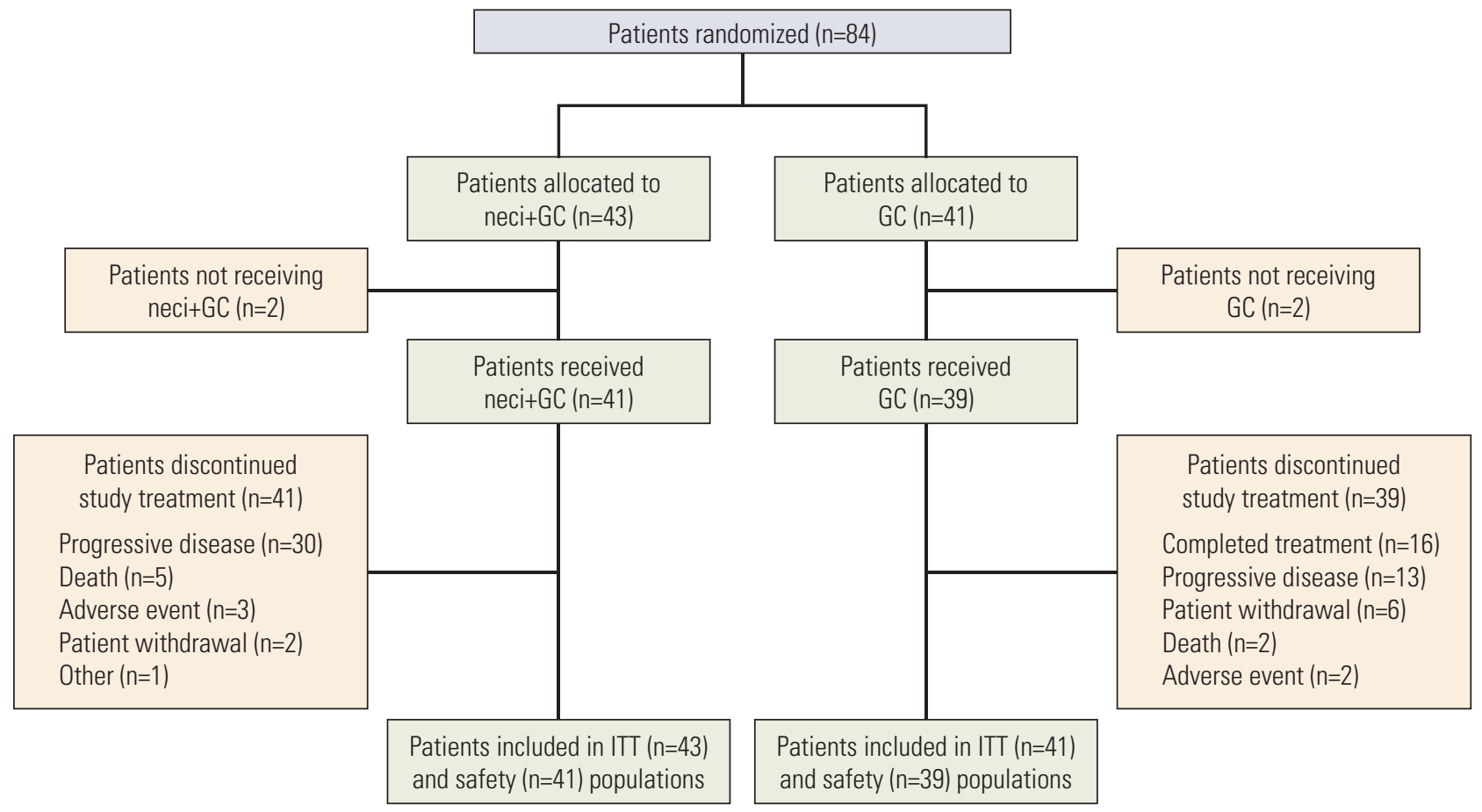

Fig. 1. Patient flow for EA patients enrolled in the SQUIRE study. Note that patients in the neci+GC arm who had not progressed at the end of the induction phase could continue to receive single-agent necitumumab in the continuation phase until radiographic documentation of disease progression, unacceptable toxicity, or withdrawal of consent. EA, East Asian; neci+GC, necitumumab plus gemcitabine and cisplatin; GC, gemcitabine and cisplatin; ITT, intent-to-treat.

constituted the ITT population, while 80 received the study drug (neci+GC, 41; GC, 39) and constituted the safety population (Fig. 1). In the non-EA subpopulation, 1,009 patients were randomized to treatment (neci+GC, 502; GC, 507) and constituted the ITT population, while 999 patients received the study drug (neci+GC, 497; GC, 502) and constituted the safety population.

\section{Demographic and baseline clinical characteristics}

In the EA subpopulation, the neci+GC and GC arms were generally balanced for baseline characteristics and baseline risk factors (Table 1). However, the proportion of patients with a history of hypertension was numerically higher in the neci+GC arm (16/41 patients, 39.0\%) than the GC arm (8/39 patients, 20.5\%). There were some differences in baseline characteristics between the EA and non-EA subpopulations, such as the percentage of patients aged $\geq 65$ years and the percentage who were non-smokers being higher in the EA subpopulation. However, the baseline characteristics of the EA subpopulation were generally consistent with those of the non-EA subpopulation (Table 1).
The proportions of patients with EGFR-expressing tumors were similar between the EA and non-EA subpopulations. The EGFR H-score was determined for $76 / 84$ patients in the EA subpopulation, while $70 / 76$ patients $(92.1 \%)$ had an $\mathrm{H}$-score $>0$ (neci+GC, 34; GC, 36). The EGFR H-score was determined for $895 / 1,009$ patients in the non-EA subpopulation and $854 / 895$ patients $(95.4 \%)$ had an $\mathrm{H}$-score $>0$ (neci+GC, 422; GC, 432).

\section{Exposure to chemotherapy and necitumumab}

The median number of cycles of gemcitabine and cisplatin was six (IQR, 4 to 6) for both drugs in the neci+GC arm and six (IQR, 2 to 6) and five (IQR, 2 to 6), respectively, in the GC $\mathrm{arm}$. The respective median relative dose intensities of gemcitabine and cisplatin were $80.3 \%$ and $90.0 \%$ in the neci+GC arm and $90.0 \%$ and $97.7 \%$ in the GC arm. In the neci+GC arm, the median number of cycles of necitumumab was seven (IQR, 4 to 9) and the median relative dose intensity of necitumumab was $90.9 \%$. Exposure to chemotherapy and necitumumab was similar between the EA and non-EA subpopulations (data not shown). 
Table 1. Patient demographics and baseline disease characteristics

\begin{tabular}{|c|c|c|c|c|}
\hline \multirow{2}{*}{ Characteristic } & \multicolumn{2}{|c|}{ EA subpopulation } & \multicolumn{2}{|c|}{ Non-EA subpopulation } \\
\hline & $\mathrm{Neci}+\mathrm{GC}(\mathrm{n}=43)$ & GC $(n=41)$ & $\mathrm{Neci}+\mathrm{GC}(\mathrm{n}=502)$ & $\mathrm{GC}(\mathrm{n}=507)$ \\
\hline Male sex & $38(88.4)$ & $35(85.4)$ & $412(82.1)$ & $423(83.4)$ \\
\hline Age, median (range, yr) & $65.0(47-76)$ & $64.0(39-79)$ & $62.0(32-84)$ & $62.0(32-86)$ \\
\hline \multicolumn{5}{|l|}{ Age group (yr) } \\
\hline$<65$ & $21(48.8)$ & $23(56.1)$ & $311(62.0)$ & $317(62.5)$ \\
\hline$\geq 65$ & $22(51.2)$ & $18(43.9)$ & $191(38.0)$ & $190(37.5)$ \\
\hline \multicolumn{5}{|l|}{ ECOG PS } \\
\hline 0 & $9(20.9)$ & $8(19.5)$ & $155(30.9)$ & $172(33.9)$ \\
\hline 1 & $33(76.7)$ & $31(75.6)$ & $299(59.6)$ & $289(57.0)$ \\
\hline 2 & $1(2.3)$ & $2(4.9)$ & $48(9.6)$ & $45(8.9)$ \\
\hline \multicolumn{5}{|l|}{ Smoking history $^{\text {b) }}$} \\
\hline Non-smoker & $5(11.6)$ & $5(12.2)$ & $21(4.2)$ & $22(4.3)$ \\
\hline Ex-light smoker & $3(7.0)$ & $4(9.8)$ & $15(3.0)$ & $22(4.3)$ \\
\hline Smoker & $35(81.4)$ & $32(78.0)$ & $465(92.6)$ & $463(91.3)$ \\
\hline
\end{tabular}

Values are presented as number (\%) unless otherwise indicated. EA, East Asian; neci+GC, necitumumab plus gemcitabine and cisplatin; GC, gemcitabine and cisplatin; ECOG, Eastern Cooperative Oncology Group; PS, performance status. ${ }^{\text {al In the }}$ GC arm (non-EA subpopulation), one patient with ECOG PS 3 in the electronic case report form (entered as ECOG PS 1 in the interactive voice response system) was randomized in the study; this patient did not receive treatment, ${ }^{\mathrm{b}}$ Information on smoking history was missing for one patient in the neci+GC arm (non-EA subpopulation).

Table 2. Summary of efficacy endpoints

\begin{tabular}{|c|c|c|c|c|}
\hline \multirow{2}{*}{ Characteristic } & \multicolumn{2}{|c|}{ EA subpopulation } & \multicolumn{2}{|c|}{ Non-EA subpopulation } \\
\hline & Neci+GC $(n=43)$ & $\mathrm{GC}(\mathrm{n}=41)$ & Neci+GC (n=502) & $\mathrm{GC}(\mathrm{n}=507)$ \\
\hline OS, median $(95 \% \mathrm{CI}, \mathrm{mo})$ & $12.0(7.3-15.2)$ & $12.2(5.5-14.7)$ & $11.5(10.5-12.6)$ & $9.8(8.8-11.1)$ \\
\hline $\operatorname{HR}(95 \% \mathrm{CI})^{\mathrm{a})}$ & \multicolumn{2}{|c|}{$0.805(0.484-1.341)$} & \multicolumn{2}{|c|}{$0.839(0.730-0.964)$} \\
\hline PFS, median $(95 \%$ CI, mo) & $5.6(4.7-6.4)$ & $5.3(2.8-5.6)$ & $5.7(5.6-6.0)$ & $5.5(4.8-5.6)$ \\
\hline $\operatorname{HR}(95 \% \mathrm{CI})^{\mathrm{a})}$ & \multicolumn{2}{|c|}{$0.720(0.439-1.180)$} & \multicolumn{2}{|c|}{$0.862(0.749-0.993)$} \\
\hline TTF, median (95\% CI, mo) & $5.5(4.2-5.7)$ & $3.7(1.6-5.3)$ & $4.3(4.2-4.8)$ & $3.6(3.2-4.1)$ \\
\hline $\operatorname{HR}(95 \% \mathrm{CI})^{\mathrm{a})}$ & \multicolumn{2}{|c|}{$0.684(0.434-1.077)$} & \multicolumn{2}{|c|}{$0.858(0.757-0.974)$} \\
\hline Complete response, $\mathrm{n}(\%)$ & 0 & 0 & 0 & $3(0.6)$ \\
\hline Partial response, $\mathrm{n}(\%)$ & $16(37.2)$ & $12(29.3)$ & $154(30.7)$ & $143(28.2)$ \\
\hline Stable disease, $\mathbf{n}(\%)$ & $19(44.2)$ & $15(36.6)$ & $257(51.2)$ & $249(49.1)$ \\
\hline Progressive disease, $\mathrm{n}(\%)$ & $3(7.0)$ & $7(17.1)$ & $38(7.6)$ & $48(9.5)$ \\
\hline Not evaluable/not assessed, $\mathrm{n}(\%)$ & $5(11.6)$ & $7(17.1)$ & $53(10.6)$ & $64(12.6)$ \\
\hline ORR $(95 \% \mathrm{CI}, \%)$ & $37.2(24.4-52.1)$ & $29.3(17.6-44.5)$ & $30.7(26.8-34.8)$ & $28.8(25.0-32.9)$ \\
\hline DCR $(95 \%$ CI, \%) & $81.4(67.4-90.3)$ & $65.9(50.5-78.4)$ & $81.9(78.3-85.0)$ & $77.9(74.1-81.3)$ \\
\hline
\end{tabular}

EA, East Asian; neci+GC, necitumumab plus gemcitabine and cisplatin; GC, gemcitabine and cisplatin; OS, overall survival; $\mathrm{CI}$, confidence interval; $\mathrm{HR}$, hazard ratio; PFS, progression-free survival; TTF, time-to-treatment failure; ORR, objective response rate; $\mathrm{DCR}$, disease control rate. ${ }^{\mathrm{a}} \mathrm{Neci}+\mathrm{GC}$ vs. GC.

\section{Efficacy measures}

OS, PFS, and TTF were improved for neci+GC versus GC in both the EA and non-EA subpopulations (Table 2, Fig. 2). In the EA subpopulation, the ORR was $37.2 \%$ and $29.3 \%$ in the neci+GC and GC arms, respectively, and the DCR was $81.4 \%$ and $65.9 \%$, respectively (Table 2). In the non-EA subpopulation, the ORR was $30.7 \%$ and $28.8 \%$ in the neci+GC and GC arms, respectively, and the DCR was $81.9 \%$ and $77.9 \%$, respectively (Table 2). 
A

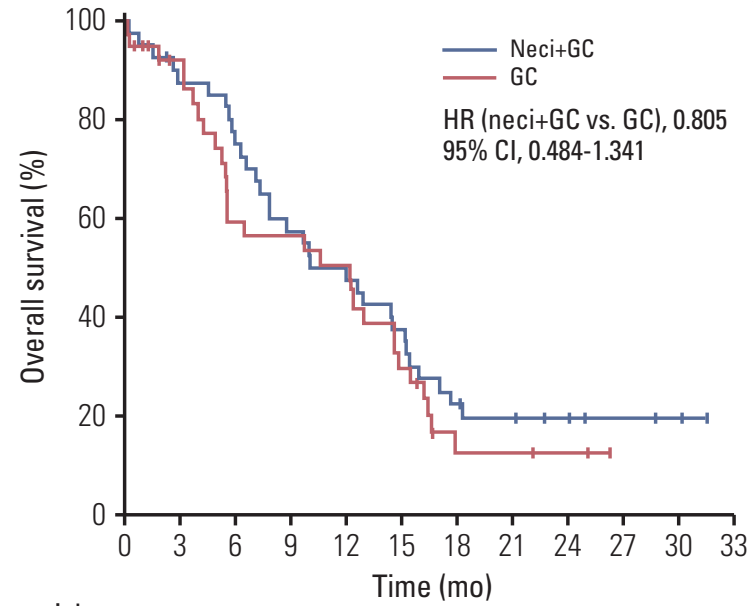

No. at risk

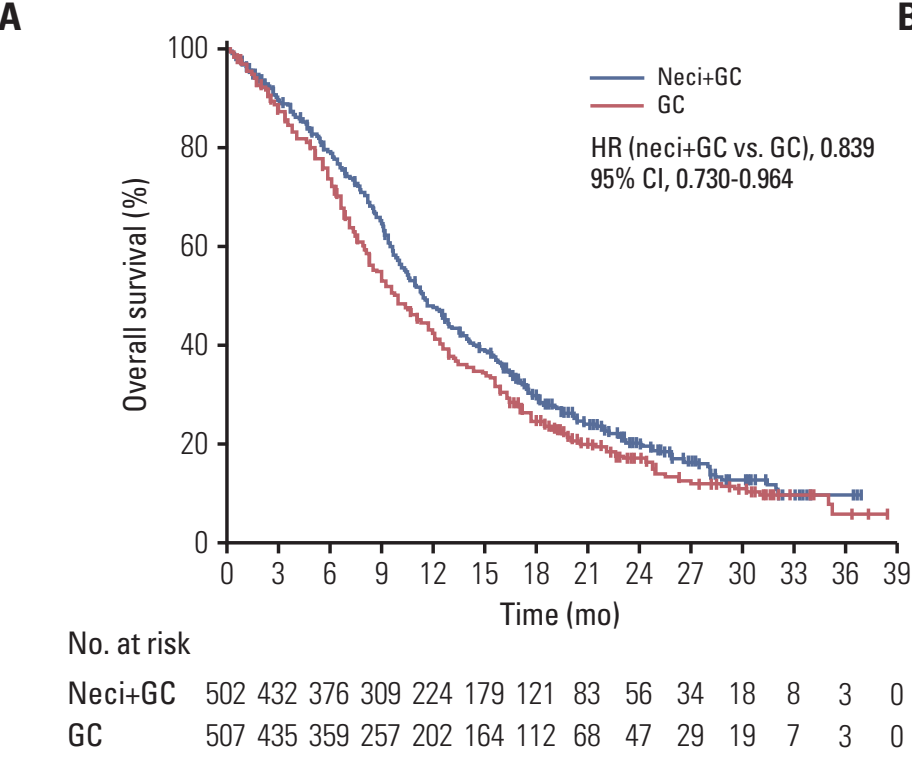

$\begin{array}{lllllllllllll}\text { Neci+GC } & 43 & 35 & 31 & 23 & 19 & 15 & 9 & 7 & 5 & 3 & 2 & 0\end{array}$ GC $\quad \begin{array}{lllllllllllll}41 & 31 & 20 & 19 & 17 & 10 & 3 & 3 & 2 & 0 & 0 & 0\end{array}$

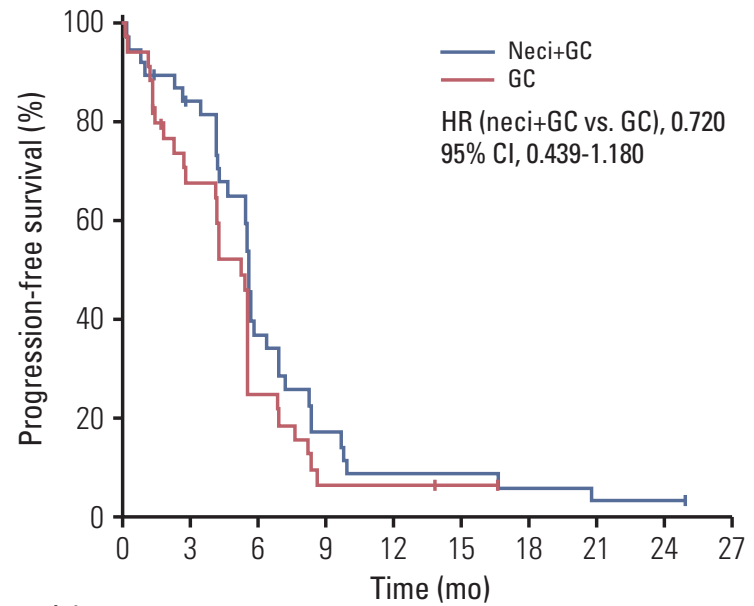

No. at risk

$\begin{array}{lllllllllll}\text { Neci+GC } & 43 & 31 & 13 & 6 & 3 & 3 & 2 & 1 & 1 & 0\end{array}$ GC $\quad \begin{array}{llllllllll}41 & 22 & 8 & 2 & 2 & 1 & 0 & 0 & 0 & 0\end{array}$
C

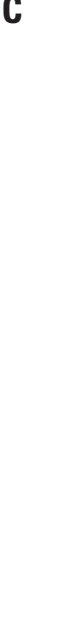

\section{No. at risk}

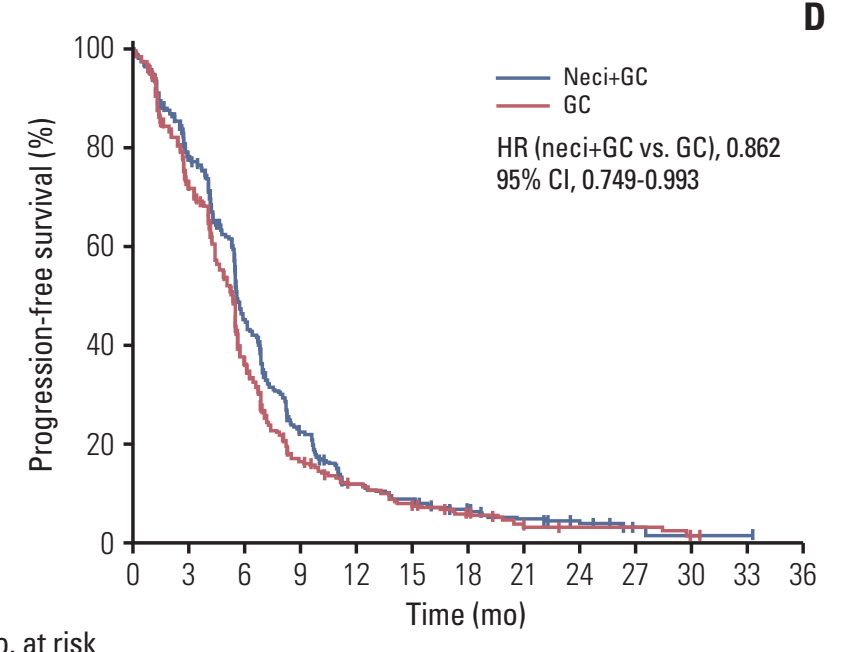

$\begin{array}{llllllllllllll}\text { Neci+GC } & 502 & 342 & 184 & 90 & 43 & 32 & 21 & 12 & 8 & 2 & 1 & 1 & 0\end{array}$ GC $\quad \begin{array}{lllllllllllll}507 & 322 & 146 & 56 & 36 & 24 & 15 & 7 & 4 & 4 & 1 & 0 & 0\end{array}$

Fig. 2. Kaplan-Meier analysis of overall survival and progression-free survival in the EA and non-EA subpopulations. Overall survival in the neci+GC and GC arms in the EA subpopulation (A) and non-EA subpopulation (B). Progression-free survival in the neci+GC and GC arms in the EA subpopulation (C) and non-EA subpopulation (D). EA, East Asian; neci+GC, necitumumab plus gemcitabine and cisplatin; GC, gemcitabine and cisplatin; $\mathrm{HR}$, hazard ratio; $\mathrm{CI}$, confidence interval.

\section{Post-study systemic therapy}

In the EA subpopulation, post-study systemic therapy was reported by a higher proportion of patients in the neci+GC arm than the GC arm (21/43 patients, $48.8 \%$ vs. $14 / 41$ patients, $34.1 \%)$, including docetaxel (34.9\% vs. $22.0 \%)$ and erlotinib (14.0\% vs. 9.8\%). In the non-EA subpopulation, post-study systemic therapy was reported by a similar proportion of patients in the neci+GC (237/502 patients, 47.2\%) and GC (231/507 patients, $45.6 \%)$ arms. Docetaxel use was 
Table 3. Summary of AEs

\begin{tabular}{|c|c|c|c|c|}
\hline \multirow{2}{*}{$\mathrm{AE}$} & \multicolumn{2}{|c|}{ EA subpopulation } & \multicolumn{2}{|c|}{ Non-EA subpopulation } \\
\hline & $\left.\mathrm{Neci}+\mathrm{GC}^{\mathrm{a}}\right)(\mathrm{n}=41)$ & $\mathrm{GC}^{\mathrm{b})}(\mathrm{n}=39)$ & $\left.\mathrm{Neci}+\mathrm{GC}^{\mathrm{a}}\right)(\mathrm{n}=497)$ & $\mathrm{GC}^{\mathrm{b})}(\mathrm{n}=502)$ \\
\hline Any AE & $41(100)$ & $37(94.9)$ & $492(99.0)$ & $492(98.0)$ \\
\hline Possibly related to neci & $30(73.2)$ & NA & $378(76.1)$ & NA \\
\hline Any SAE & $27(65.9)$ & $20(51.3)$ & $230(46.3)$ & $183(36.5)$ \\
\hline Possibly related to neci & $1(2.4)$ & NA & $31(6.2)$ & NA \\
\hline Any grade $\geq 3 \mathrm{AE}$ & $36(87.8)$ & $25(64.1)$ & $352(70.8)$ & $308(61.4)$ \\
\hline Possibly related to neci & $5(12.2)$ & NA & $84(16.9)$ & NA \\
\hline Any AE with outcome of death & $7(17.1)$ & $3(7.7)$ & 59 (11.9) & $54(10.8)$ \\
\hline Possibly related to neci & 0 & NA & $2(0.4)$ & NA \\
\hline $\begin{array}{l}\text { Any AE leading to discontinuation } \\
\text { of any study drug }\end{array}$ & $8(19.5)$ & $4(10.3)$ & $160(32.2)$ & $129(25.7)$ \\
\hline Any AE leading to discontinuation of neci & $1(2.4)$ & NA & $39(7.8)$ & NA \\
\hline
\end{tabular}

Values are presented as number (\%). AE, adverse event; EA, East Asian; neci+GC, necitumumab plus gemcitabine and cisplatin; GC, gemcitabine and cisplatin; SAE, serious adverse event; NA, not applicable. ${ }^{\text {a) }}$ Safety observation period=treatment and continuation phases, ${ }^{b}$ Safety observation period=continuation phase.

higher in the neci+GC arm than the GC arm (30.3\% vs. $23.3 \%$ ), while erlotinib use was higher in the GC arm than the neci+GC arm (14.0\% vs. $10.2 \%)$.

\section{Safety and tolerability measures}

\section{1) Adverse events}

The proportions of patients experiencing serious adverse events (SAEs), AEs of grade $\geq 3$, and AEs with an outcome of death were numerically higher for (1) EA patients treated with neci+GC than EA patients treated with GC and (2) EA patients treated with neci+GC than non-EA patients treated with neci+GC (Table 3). The proportion of patients experiencing AEs possibly related to necitumumab was similar for the EA and non-EA subpopulations (Table 3). For patients treated with GC, the proportion of patients experiencing SAEs was higher in the EA subpopulation than in the nonEA subpopulation, while the proportions of patients experiencing AEs of grade $\geq 3$ and AEs with an outcome of death were similar in the EA and non-EA subpopulations (Table 3). AEs leading to the discontinuation of any study drug were reported more frequently in the neci+GC arm than the GC arm in both the EA and non-EA subpopulations (Table 3).

\section{2) AEs of interest}

In the EA subpopulation, expected EGFR-associated toxicities such as rash, hypomagnesemia, and venous thromboembolic events were reported more frequently in the neci+GC arm than the GC arm (Table 4). In particular, 80.5\% of EA patients receiving neci+GC reported skin rash of any grade compared with $17.9 \%$ of EA patients receiving GC (Table 4). In general, the profiles of the AEs of interest were similar between the EA and non-EA subpopulations (Table 4). There were some numerical differences between the neci+GC arms in the EA and non-EA subpopulations. Specifically, neutropenia and thrombocytopenia were reported more frequently, and hypomagnesemia less frequently, in the neci+GC arm in the EA subpopulation than the non-EA subpopulation.

\section{Discussion}

This post hoc subgroup analysis of the SQUIRE study showed that addition of an agent targeting the EGFR to platinum-based doublet chemotherapy resulted in a survival benefit for EA patients with advanced squamous NSCLC. The EA patients who received neci+GC also experienced improvements in PFS, TTF, ORR, and DCR compared with those receiving GC, and these improvements in efficacy were accompanied by an acceptable safety profile. The efficacy and safety results observed for neci+GC versus GC in the EA subpopulation were consistent with those observed in the non-EA subpopulation of the SQUIRE study, which constituted a large, predominantly Caucasian, multinational patient population. The results of this subgroup analysis suggest that necitumumab, in combination with gemcitabine and cisplatin chemotherapy, may provide an additional first- 
Table 4. Adverse events of interest

\begin{tabular}{|c|c|c|c|c|c|c|c|c|}
\hline \multirow{3}{*}{$\begin{array}{l}\text { Adverse event } \\
\text { of interest }\end{array}$} & \multicolumn{4}{|c|}{ EA subpopulation } & \multicolumn{4}{|c|}{ Non-EA subpopulation } \\
\hline & \multicolumn{2}{|c|}{ Neci+GC (n=41) } & \multicolumn{2}{|c|}{ GC $(n=39)$} & \multicolumn{2}{|c|}{ Neci+GC (n=497) } & \multicolumn{2}{|c|}{$G C(n=502)$} \\
\hline & Any grade & Grade $\geq 3$ & Any Grade & Grade $\geq 3$ & Any grade & Grade $\geq 3$ & Any grade & Grade $\geq 3$ \\
\hline Neutropenia & $25(61.0)$ & $15(36.6)$ & $20(51.3)$ & $12(30.8)$ & $210(42.3)$ & $116(23.3)$ & $228(45.4)$ & $137(27.3)$ \\
\hline Febrile neutropenia & $2(4.9)$ & $1(2.4)$ & $1(2.6)$ & $1(2.6)$ & $4(0.8)$ & $3(0.6)$ & $7(1.4)$ & $6(1.2)$ \\
\hline Anemia & $16(39.0)$ & $5(12.2)$ & $14(35.9)$ & $6(15.4)$ & $209(42.1)$ & $52(10.5)$ & $234(46.6)$ & $53(10.6)$ \\
\hline Thrombocytopenia & $14(34.1)$ & $5(12.2)$ & $13(33.3)$ & $5(12.8)$ & $103(20.7)$ & $50(10.1)$ & $133(26.5)$ & $53(10.6)$ \\
\hline Fatigue & $15(36.6)$ & 0 & $20(51.3)$ & $2(5.1)$ & $214(43.1)$ & $39(7.8)$ & $210(41.8)$ & $36(7.2)$ \\
\hline Hypomagnesemia & $7(17.1)$ & $1(2.4)$ & $5(12.8)$ & 0 & $161(32.4)$ & $49(9.9)$ & $80(15.9)$ & $6(1.2)$ \\
\hline Skin rash & $33(80.5)$ & $2(4.9)$ & 7 (17.9) & 0 & $377(75.9)$ & $36(7.2)$ & $48(9.6)$ & $2(0.4)$ \\
\hline Interstitial lung disease & $2(4.9)$ & $1(2.4)$ & $1(2.6)$ & 0 & $3(0.6)$ & $1(0.2)$ & $3(0.6)$ & $3(0.6)$ \\
\hline $\begin{array}{l}\text { Venous thromboembolic } \\
\text { events }\end{array}$ & $5(12.2)$ & $1(2.4)$ & $2(5.1)$ & $1(2.6)$ & $44(8.9)$ & $26(5.2)$ & $27(5.4)$ & $13(2.6)$ \\
\hline $\begin{array}{l}\text { Arterial thromboembolic } \\
\text { events }\end{array}$ & $4(9.8)$ & $2(4.9)$ & $3(7.7)$ & $1(2.6)$ & $25(5.0)$ & $19(3.8)$ & $18(3.6)$ & $10(2.0)$ \\
\hline
\end{tabular}

Values are presented as number (\%). EA, East Asian; neci+GC, necitumumab plus gemcitabine and cisplatin; GC, gemcitabine and cisplatin.

line treatment option for EA patients with advanced squamous NSCLC, a tumor type for which new treatments have been scarce over the past decade [1].

Overall, the efficacy results for the EA subpopulation in the SQUIRE study were consistent with those for the non-EA subpopulation and for the overall SQUIRE study population [9]. Taking the small number of EA patients and the limitations of this post hoc analysis into account, improvements in the OS and PFS for neci+GC versus GC for the EA subpopulation were in a similar range to those for the non-EA subpopulation and the overall SQUIRE study population [9]. The HRs for OS and PFS for the EA subpopulation were in the same direction as, and overlapped with, those of the nonEA subpopulation and the overall SQUIRE study population [9]. These findings indicate that the true effect of neci+GC in the EA subpopulation may not be different from the treatment effect observed in the non-EA subpopulation or the overall SQUIRE study population; namely, a reduction in risk of death and disease progression. However, it should be noted that the use of post-study systemic therapy in the EA subpopulation was higher in the neci+GC arm than the GC $\operatorname{arm}(48.8 \%$ vs. $34.1 \%)$. An effect of post study therapy on OS cannot be ruled out.

Overall, the safety results for the EA subpopulation in the SQUIRE study were consistent with those for the non-EA subpopulation and for the overall SQUIRE study population [9]. As observed for the overall SQUIRE study population [9], the addition of necitumumab to gemcitabine and cisplatin chemotherapy was associated with an increase in grade 3 or higher AEs and in expected EGFR-associated toxicities [17-19], such as rash, hypomagnesemia, and venous thromboembolic events. There were numerical differences in the incidence of some types of AEs (SAEs, AEs of grade $\geq 3$, AEs with an outcome of death, neutropenia, thrombocytopenia, hypomagnesemia) between the neci+GC arms in the EA and non-EA subpopulations, which may be a result of the small number of patients in the EA subpopulation. Moreover, when interpreting these safety results, it should be noted that (1) some risk factors (e.g., history of hypertension) were not balanced between treatment arms and (2) the safety observation period for the neci+GC arm included the treatment and continuation phases and was therefore longer than the safety observation period for the GC arm, which only included the induction phase. Nevertheless, the safety results observed in the neci+GC arm in the EA subpopulation represent an acceptable safety profile for a first-line treatment regimen for advanced squamous NSCLC. The median relative dose intensities of gemcitabine $(80.3 \%)$ and cisplatin $(90.0 \%)$ in the neci+GC arm in the EA subpopulation are also evidence that the addition of necitumumab to gemcitabine and cisplatin chemotherapy is well tolerated in this study population.

A few treatments have recently shown clinical benefit in patients with advanced squamous NSCLC. These include nab-paclitaxel, an albumin-bound formulation of paclitaxel, nivolumab and pembrolizumab, monoclonal antibody inhibitors of programmed cell death 1 protein, and cetuximab. A study of nab-paclitaxel plus carboplatin compared 
with solvent-based paclitaxel plus carboplatin as first-line treatment in patients with advanced NSCLC showed a higher response rate ( $41 \%$ vs. $24 \%$ ) and longer median OS (10.7 vs. 9.5 months) with nab-paclitaxel versus solventbased paclitaxel in the subgroup of 450 patients with squamous NSCLC. Specifically, the response rate (26\% vs. $25 \%$ ) and median OS (13.1 months vs. 13.0 months) were similar between the two treatment regimens in the subgroup of 602 patients with nonsquamous NSCLC [20]. In a study of 272 patients with previously treated squamous NSCLC, nivolumab significantly improved OS compared with docetaxel (9.2 months vs. 6.0 months) [21]. The chimeric EGFR antibody cetuximab interacts with a similar EGFR epitope to necitumumab [22]. The survival benefit observed in response to cetuximab treatment in the subgroup of patients with squamous NSCLC in the FLEX study [16] was confirmed in a meta-analysis of four open-label randomized studies, which included the FLEX study (HR, 0.77; 95\% CI, 0.64 to 0.93) [23]. Previous studies have shown that targeting the EGFR with EGFR TKIs has not benefited patients with squamous NSCLC [1,2]. However, these cetuximab studies, along with the SQUIRE study [9], indicate that targeting the EGFR with a monoclonal antibody is a promising approach for the treatment of patients with squamous NSCLC.

This subgroup analysis of EA patients enrolled in the SQUIRE study was limited by the small sample size, which led to insufficient power to demonstrate a statistically significant difference between treatment arms in the EA subpopulation. Other limitations included the post hoc nature of the analysis and the lack of formal comparisons between the EA and non-EA subpopulations. Moreover, some risk factors (e.g., history of hypertension) were not balanced between treatment arms. A strength of the analysis was that the baseline demographics and disease characteristics of the EA subpopulation were similar to the overall population and reflective of a typical squamous NSCLC patient population, with the majority of patients being male and smokers.

Although limited by the small sample size and post hoc nature of the analysis, the efficacy of neci+GC for the EA patients in the SQUIRE study was consistent with that observed for the non-EA patients, with improvements in OS, PFS, and TTF. In addition, the overall safety data for the EA patients in the SQUIRE study were consistent with those for the non-EA patients and represent an acceptable safety profile. Taken together, these findings suggest that the addition of necitumumab to platinum-based doublet chemotherapy may offer a survival advantage and favorable benefit/risk for EA patients with advanced squamous NSCLC.

\section{Conflicts of Interest}

This study was sponsored by Eli Lilly, the manufacturer/ licensee of necitumumab. Medical writing assistance was provided by Justine Southby, PhD, CMPP, and Luke Carey, PhD, of ProScribeEnvision Pharma Group, and was funded by Eli Lilly. ProScribe's services complied with international guidelines for Good Publication Practice (GPP3).

Eli Lilly was involved in the study design, data collection, data analysis, and preparation of the manuscript.

V.S., H.D., T.P., and M.O. are employees of and own stock in Eli Lilly. T.P. is on the Board of Directors of Eli Lilly and Company (India) Pvt. Ltd. K.P. has an advisory role with Eli Lilly. E.K.C., M.B., M.-J.A., S.T., and E.-K.S. have no conflicts of interest to declare.

\section{References}

1. Perez-Moreno P, Brambilla E, Thomas R, Soria JC. Squamous cell carcinoma of the lung: molecular subtypes and therapeutic opportunities. Clin Cancer Res. 2012;18:2443-51.

2. Gandara DR, Hammerman PS, Sos ML, Lara PN Jr, Hirsch FR. Squamous cell lung cancer: from tumor genomics to cancer therapeutics. Clin Cancer Res. 2015;21:2236-43.

3. Gentzler RD, Johnson ML. Complex decisions for first-line and maintenance treatment of advanced wild-type non-small cell lung cancer. Oncologist. 2015;20:299-306.

4. Pirker R, Pereira JR, Szczesna A, von Pawel J, Krzakowski M, Ramlau R, et al. Cetuximab plus chemotherapy in patients with advanced non-small-cell lung cancer (FLEX): an openlabel randomised phase III trial. Lancet. 2009;373:1525-31.

5. Sgambato A, Casaluce F, Maione P, Rossi A, Ciardiello F, Gridelli C. Cetuximab in advanced non-small cell lung cancer
(NSCLC): the showdown? J Thorac Dis. 2014;6:578-80.

6. Hirsch FR, Varella-Garcia M, Bunn PA Jr, Di Maria MV, Veve $\mathrm{R}$, Bremmes RM, et al. Epidermal growth factor receptor in non-small-cell lung carcinomas: correlation between gene copy number and protein expression and impact on prognosis. J Clin Oncol. 2003;21:3798-807.

7. Dacic S, Flanagan M, Cieply K, Ramalingam S, Luketich J, Belani C, et al. Significance of EGFR protein expression and gene amplification in non-small cell lung carcinoma. Am J Clin Pathol. 2006;125:860-5.

8. Greillier L, Tomasini P, Barlesi F. Necitumumab for non-small cell lung cancer. Expert Opin Biol Ther. 2015;15:1231-9.

9. Thatcher N, Hirsch FR, Luft AV, Szczesna A, Ciuleanu TE, Dediu M, et al. Necitumumab plus gemcitabine and cisplatin versus gemcitabine and cisplatin alone as first-line therapy in 
patients with stage IV squamous non-small-cell lung cancer (SQUIRE): an open-label, randomised, controlled phase 3 trial. Lancet Oncol. 2015;16:763-74.

10. Sekine I, Yamamoto N, Nishio K, Saijo N. Emerging ethnic differences in lung cancer therapy. Br J Cancer. 2008;99:1757-62.

11. Iwasaki M, Hinotsu S, Katsura J. Clinical trials and approval of anti-cancer agents. Jpn J Clin Oncol. 2010;40 Suppl 1:i65-9.

12. Paez JG, Janne PA, Lee JC, Tracy S, Greulich H, Gabriel S, et al. EGFR mutations in lung cancer: correlation with clinical response to gefitinib therapy. Science. 2004;304:1497-500.

13. Shigematsu H, Lin L, Takahashi T, Nomura M, Suzuki M, Wistuba II, et al. Clinical and biological features associated with epidermal growth factor receptor gene mutations in lung cancers. J Natl Cancer Inst. 2005;97:339-46.

14. O'Donnell PH, Dolan ME. Cancer pharmacoethnicity: ethnic differences in susceptibility to the effects of chemotherapy. Clin Cancer Res. 2009;15:4806-14.

15. Edge S, Byrd DR, Compton CC, Fritz AG, Greene FL, Trotti A. AJCC cancer staging manual. 7th ed. New York: Springer; 2010.

16. Pirker R, Pereira JR, von Pawel J, Krzakowski M, Ramlau R, Park K, et al. EGFR expression as a predictor of survival for first-line chemotherapy plus cetuximab in patients with advanced non-small-cell lung cancer: analysis of data from the phase 3 FLEX study. Lancet Oncol. 2012;13:33-42.

17. Segaert S, Van Cutsem E. Clinical signs, pathophysiology and management of skin toxicity during therapy with epidermal growth factor receptor inhibitors. Ann Oncol. 2005;16:1425-33.

18. Chen P, Wang L, Li H, Liu B, Zou Z. Incidence and risk of hypomagnesemia in advanced cancer patients treated with cetuximab: a meta-analysis. Oncol Lett. 2013;5:1915-20.

19. Petrelli F, Cabiddu M, Borgonovo K, Barni S. Risk of venous and arterial thromboembolic events associated with antiEGFR agents: a meta-analysis of randomized clinical trials. Ann Oncol. 2012;23:1672-9.

20. Socinski MA, Bondarenko I, Karaseva NA, Makhson AM, Vynnychenko I, Okamoto I, et al. Weekly nab-paclitaxel in combination with carboplatin versus solvent-based paclitaxel plus carboplatin as first-line therapy in patients with advanced non-small-cell lung cancer: final results of a phase III trial. J Clin Oncol. 2012;30:2055-62.

21. Brahmer J, Reckamp KL, Baas P, Crino L, Eberhardt WE, Poddubskaya E, et al. Nivolumab versus docetaxel in advanced squamous-cell non-small-cell lung cancer. N Engl J Med. 2015;373:123-35.

22. Li S, Kussie P, Ferguson KM. Structural basis for EGF receptor inhibition by the therapeutic antibody IMC-11F8. Structure. 2008;16:216-27.

23. Pujol JL, Pirker R, Lynch TJ, Butts CA, Rosell R, Shepherd FA, et al. Meta-analysis of individual patient data from randomized trials of chemotherapy plus cetuximab as first-line treatment for advanced non-small cell lung cancer. Lung Cancer. 2014;83:211-8. 